\section{Pierre R. Blanquet}

\section{ADRESSE}

P.R. Blanquet : directeur de recherche à l'Inserm. Inserm U. 161, pharmacologie du système nerveux, 2, rue d'Alésia, 75014 Paris, France.

$m / s n^{\circ} 3$, vol. 12 , mars 96

\title{
Les signaux des FGF: un mécanisme qui commence à être déchiffré
}

Les FGF (fibroblast growth factors) sont des peptides multifonctionnels. Ils appartiennent à une famille qui compte actuellement neuf membres. Leur système de signalisation commence à être déchiffré; il implique principalement trois catégories de composés moléculaires: une pléiade d'isoformes de récepteurs à activité tyrosine kinase et des corécepteurs qui sont des protéoglycanes à sulfate d'héparane; de nombreux effecteurs et seconds messagers impliqués dans la signalisation des facteurs de croissance et des neurotrophines; des cibles nucléaires capables d'être directement activées par des FGF endogènes et des FGF exogènes internalisés.

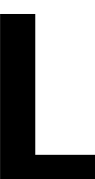

a compréhension des mécanismes de signalisation des facteurs de croissance FGF ( $f$ broblast growth factors) devrait avoir un impact important sur la médecine des affections majeures telles que le cancer, la polyarthrite rhumatoïde, certaines neurodégénérescences, l'angiogenèse et plusieurs syndromes d'origine génétique $(\mathrm{m} / \mathrm{s}$ $n^{\circ} 8 / 9$, vol. 10 , p.936; $n^{\circ} 11$, vol. 11 , p. $1163 ; n^{\circ} 12$, vol. 11, p. 1768) [1-4]. Trois étapes ont stimulé les recherches dans ce domaine: d'une part, la découverte que ces facteurs activent des récepteurs à activité tyrosine kinase codés par plusieurs gènes [5]; d'autre part, l'observation qu'un cofacteur glycanique (sulfate d'héparane ou héparine) paraît être nécessaire pour assurer l'activation de ces récepteurs [4]; enfin, la découverte que $\mathrm{FGF}_{2}$ peut être directement transféré dans le noyau, de façon concomitante au déclenchement du cycle cellulaire de prolifération [6]. A l'heure actuelle, on connaît neuf FGF et trois familles de composés impliquées dans leur réception à la surface cellulaire : un récepteur de forte affinité, sans activité tyrosine kinase, appelé CFR (chicken cysteine-rich fibroblast growth factor receptor); des récepteurs de forte affinité qui possèdent une activité tyrosine kinase, codés par quatre gènes (FGFR) ; et des protéoglycanes à sulfate d'héparane (HSPG) associés à la matrice extracellulaire et à la membrane plasmique [4]. En outre, on admet que les FGF sont capables de déclencher deux processus de signalisation: l'activation, par l'intermédiaire de leurs récepteurs, d'une myriade de protéines transductionnelles et de seconds messagers, et l'activation directe de plusieurs cibles nucléaires. 


\section{Les récepteurs: un foisonnement de formes multigéniques}

Le CFR est constitué d'une importante région extracellulaire, riche en cystéines, possédant cinq sites potentiels de N-glycosylation, et d'un petit domaine intracellulaire de treize acides aminés [7] (figure 1). Ce curieux récepteur est encore l'unique représentant de sa famille ; on ne possède aucune donnée précise sur son fonctionnement. Dernièrement, des auteurs ont suggéré qu'il pourrait être impliqué dans l'adhérence cellulaire [8]. En revanche, une multitude d'informations ont été obtenues sur les FGFR [5, 9-11]. Les gènes des FGFR peuvent coder pour des formes transmembranaires longues qui possèdent un domaine tyrosine kinase intracytoplasmique et une région extracellulaire formée de trois structures pseudo-immunoglobulines (Ig) et de six à neuf sites potentiels de Nglycosylation. Cependant, leur propriété la plus remarquable est de pouvoir aussi engendrer un nombre considérable de variants (théoriquement une centaine), vraisemblablement par épissage alternatif. Pour l'instant, une soixantaine de ces isoformes ont été caractérisées. Quelques variants de $\mathrm{FGFR}_{1}, \mathrm{FGFR}_{2}$ et $\mathrm{FGFR}_{3}$ sont uniquement constitués d'une région extracellulaire et sont solubles. Il s'agit d'iso-

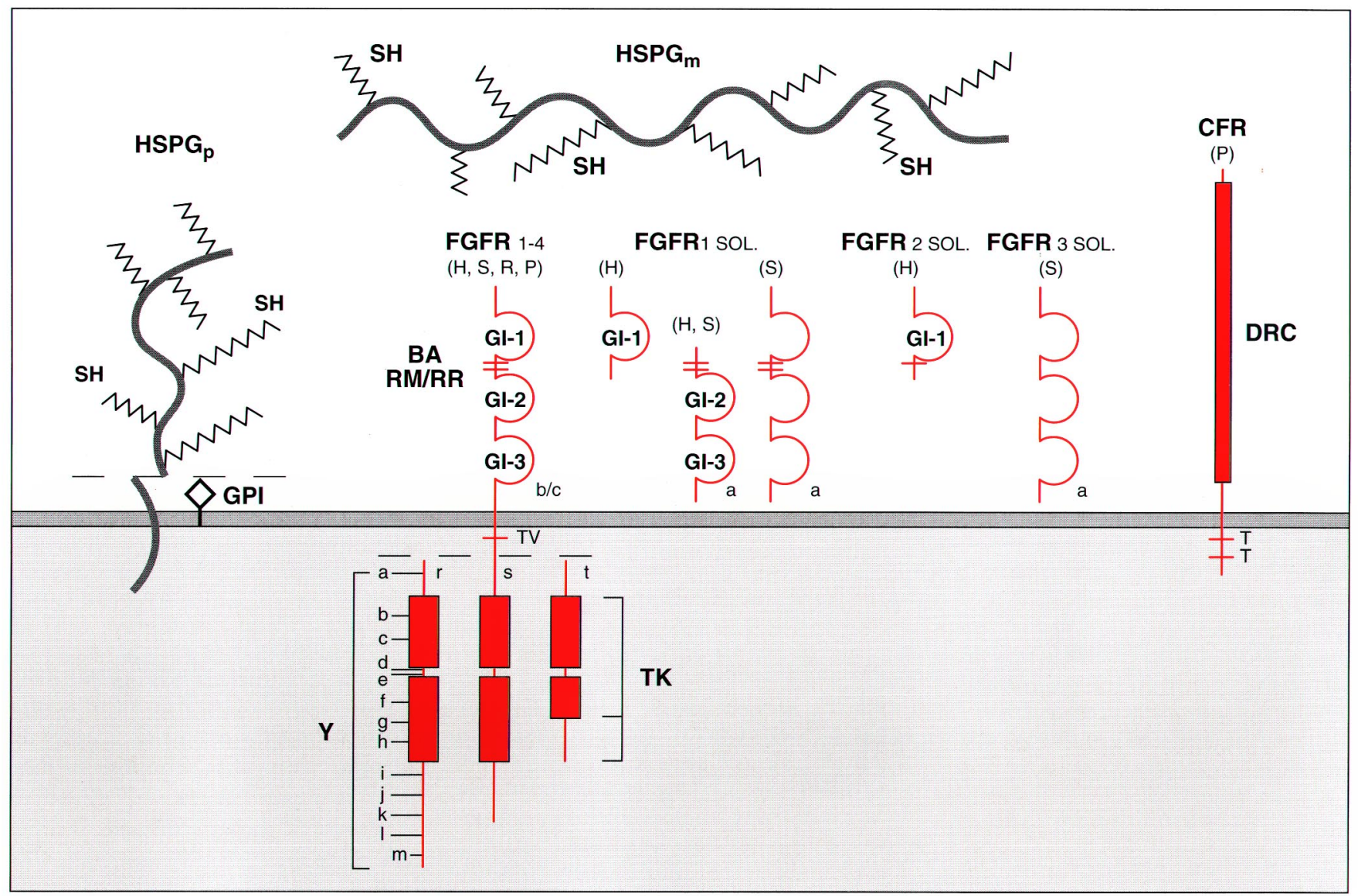

Figure 1. Représentation schématique des différents composés impliqués dans la réception des FGF à la surface cel-

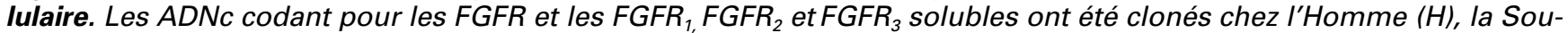
ris (S), le Rat $(R)$ et le Poulet $(P)$; I'ADNc codant pour le CFR (chicken cysteine-rich fibroblast growth-factor receptor) a

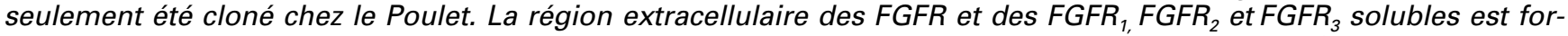
mée de 1, 2 ou 3 structures pseudo-immunoglobulines (lg) et contient trois domaines qui peuvent faire l'objet de modifications de type insertion/délétion : une "boîte" acide (BA) ; un dipeptide RM (Arg-Met) ou RR (Arg-Arg) ; la moitié C-terminale de lg-3 codée par les exons épissés $a, b$ ou c. La région intracellulaire des FGFR possède un dipeptide TV (Thr-Val) qui est délété ou non, un domaine tyrosine kinase (TK) qui est fonctionnel (variants $r$ et s) ou non (variants tronqués $t)$ et des tyrosines $\left(Y_{a-m}\right)$ qui sont distribuées de manière différente selon les classes ou les sous-classes des récepteurs. Le CFR possède une région extracellulaire qui contient un domaine riche en cystéines (DRC: 16 sous-unités de 67 cystéines) et une région intracellulaire qui possède 2 résidus Thr (T) phosphorylables. Les HSPG (protéoglycanes à sulfate d'héparane) associés à la matrice extracellulaire (HSPG ${ }_{m}$ ) et les HSPG associés à la membrane plasmique $\left(H S P G_{p}\right)$ sont constitués d'un corps protéique et de plusieurs chaînes glycaniques de sulfate d'héparane. Le corps protéique des HSPG peut être transmembranaire (cas des syndécanes) ou associé à la membrane par l'intermédiaire d'un groupement glycosyl-phosphatidylinositol (GPI) (cas des glypicanes). 
formes de $\mathrm{FGFR}_{1}$ et $\mathrm{FGFR}_{3}$ possédant deux ou trois structures Ig, dont la moitié C-terminale de la structure Ig-3 C-terminale est codée par un exon épissé a, et d'isoformes de $\mathrm{FGFR}_{1}$ et $\mathrm{FGFR}_{2}$ formées de la structure Ig-1 amino-terminale (figure 1). Leur fonction n'est pas connue. Mais la plupart des variants sont transmembranaires et résultent de la combinaison des délétions de six domaines : une structure Ig-1 amino-terminale, présente dans tous les FGFR ; une «boîte» acide constituée de quatre à huit acides aminés (BA), présente dans $\mathrm{FGFR}_{1}$ et $\mathrm{FGFR}_{2}$; un dipeptide Arg-Met (RM) ou ArgArg (RR; chez la souris), présent dans FGFR $_{1}$; une structure Ig-3 carboxy-terminale, présente dans tous les FGFR, dont la moitié carboxy-terminale est codée par deux exons épissés b et c ; un dipeptide Thr-Val (TV), présent dans FGFR $_{1}$, FGFR $_{2}$ et FGFR $_{3}$; et un domaine intracellulaire, tronqué ou non, dans la région carboxy-terminale $(r, s, t)$. En outre, le nombre des tyrosines du domaine intracellulaire varie en fonction du récepteur et parfois de la forme variante (figure 1; Tableaux I et II). Chaque type cellulaire contient généralement plusieurs de ces variants. Par exemple, le derme de la souris contient les variants FGFR $_{1(\mathrm{c})}, \mathrm{FGFR}_{2(\mathrm{~b}, \mathrm{c})}$ et $\mathrm{FGFR}_{3(\mathrm{~b}, \mathrm{c})}$ et son épiderme possède FGFR $_{2(\mathrm{~b})}$ et $\mathrm{FGFR}_{3(\mathrm{~b})}$ [12]. De plus, la distribution relative de ces variants pa-

Tableau I

COMPARAISON DE LA STRUCTURE DES RÉGIONS EXTRACELLULAIRES DES RÉCEPTEURS FGFR HUMAINS

\begin{tabular}{|c|c|c|c|c|c|c|c|c|c|}
\hline \multirow{2}{*}{$\begin{array}{l}\text { Insertions - délétions } \\
\text { de la région } \\
\text { extracellulaire }\end{array}$} & \multicolumn{3}{|c|}{ FGFR $_{1}$} & \multicolumn{4}{|c|}{ FGFR $_{2}$} & \multirow[b]{2}{*}{ FGFR $_{3}$} & \multirow[b]{2}{*}{ FGFR $_{4}$} \\
\hline & Flg $\alpha$ & Flg $\beta$ & $\operatorname{Flg} \lambda$ & $\begin{array}{l}\text { FGFR } \\
\text { Bek }\end{array}$ & $\begin{array}{l}\text { (Bek) } \\
\text { TK 14,25 }\end{array}$ & $\begin{array}{c}\text { FGFR }_{2} \\
\text { (K-sam) }\end{array}$ & -sam) & & \\
\hline $\begin{array}{l}\text { BA } \\
\text { RN } \\
\lg -1 \\
\lg -3\end{array}$ & $\begin{array}{l}+ \\
+/- \\
+\end{array}$ & $\begin{array}{c}+ \\
+/ \\
- \\
\mathrm{B} / \mathrm{C}\end{array}$ & $\begin{array}{c}- \\
+/- \\
-\end{array}$ & $\begin{array}{c}+ \\
- \\
+/-\end{array}$ & $\begin{array}{l}+ \\
- \\
+\end{array}$ & $\begin{array}{c}+ \\
- \\
+/-\end{array}$ & $\begin{array}{c}+ \\
- \\
+/-\end{array}$ & $\begin{array}{l}- \\
- \\
+ \\
\mathrm{B} / \mathrm{c}\end{array}$ & $\begin{array}{l}- \\
- \\
+ \\
c\end{array}$ \\
\hline
\end{tabular}

La classe FGFR, encore appelée Flg (Fms-like gene), se subdivise en 3 sous-classes $\alpha$, $\beta$ et $\lambda$. La classe FGFR, se subdivise en 2 sous-classes: la sousclasse Bek (Bacterially expressed kinase), composée des récepteurs Bek proprement dits et des récepteurs TK14,25 (Tyrosine kinase, clones 14 et 25) et la sous-classe $K$-sam (Kato 3 cell-derived stomach cancer amplified gene), composée des récepteurs $K$-sam proprement dits et des récepteurs $R$ KGF (ou FGFR $F_{7}$ ). On peut remarquer que la combinaison des insertions/délétions potentielles de la région extracellulaire avec celles de la région intracellulaire (Tableau 2) autorise l'existence de plusieurs variants par sous-classe. Le symbole $+/-$ indique, soit une absence, soit une présence.

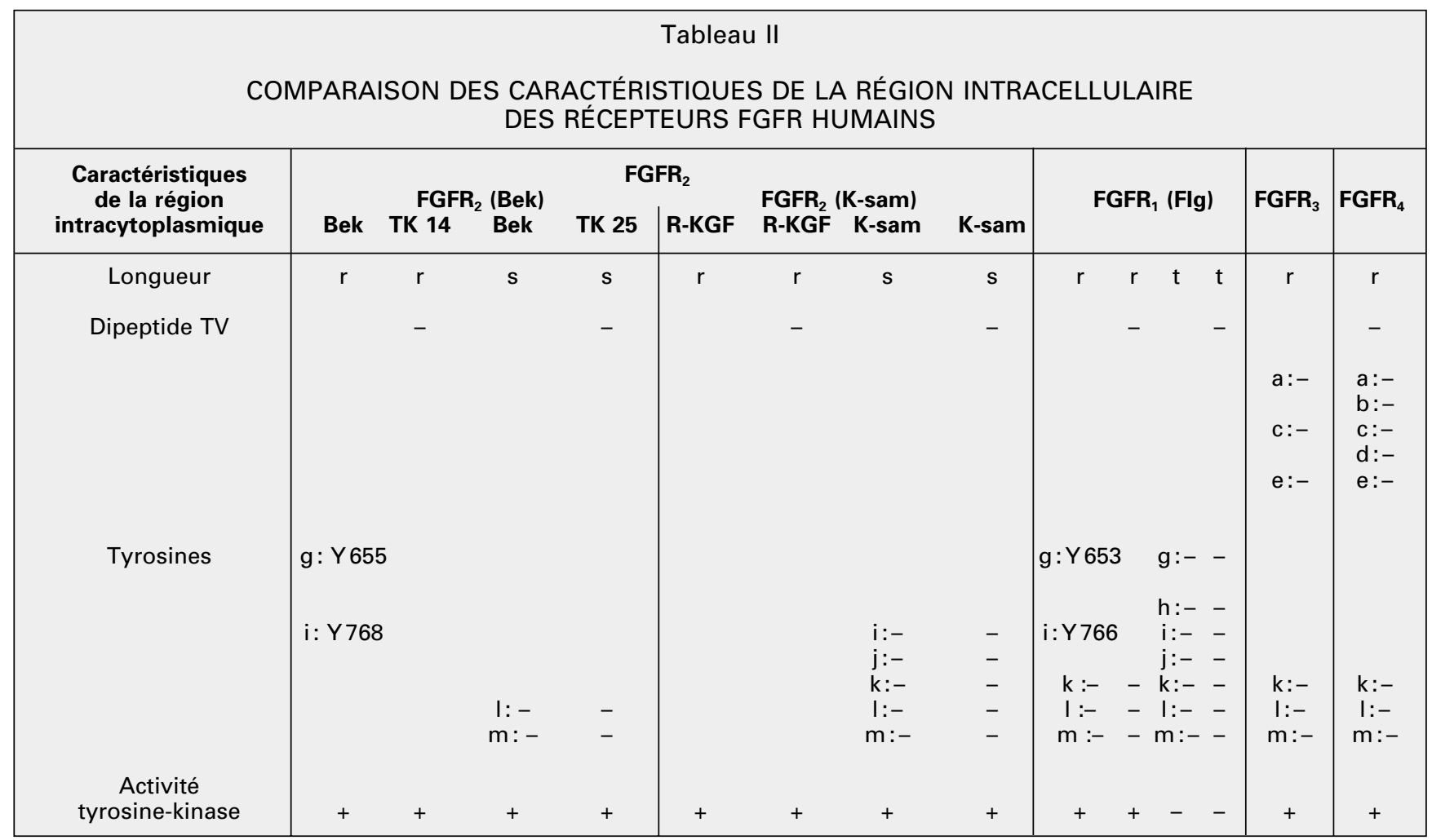

La région intracellulaire des FGFR possède trois caractéristiques structurales essentielles: une longueur variable non tronquée (r,s) ou tronquée (t), qui permet une activité tyrosine kinase (+) ou entraîne son absence (-) dans certains variants; un dipeptide TV qui est délété (-) dans certains variants; un ensemble de tyrosines (a-m) qui sont délétées (-) qui dans certains variants et dont certaines sont phosphorylées par le récepteur (cas, par exemple de $Y 655$ et $Y 768$ pour Bek et de Y653 et $Y 766$ pour la forme de FGFR, qui possède une région intracellulaire de longueur r) (voir figure 1). 


\section{RÉFÉRENCES}

1. Tobelem G. L'angiogenèse tumorale. médecine/sciences $1990 ; 6$ : 426-32.

2. Unsicker K. Growth factors in Parkinson's disease. Prog Growth Factor Res 1994 ; 5 : 7387.

3. Folkman J. The angiogenic activity of FGF and its possible implication. In: Vicki RS, ed. Growth factors: from genes to clinical application. New York: Raven Press, 1990 : 20116.

4. Fernig DG, Gallagher JT. Fibroblast growth factors and their receptors : an information network controlling tissue growth, morphogenesis and repair. Prog Growth Factor Res 1994 ; 5 : 353-77.

5. Johnson DE, Williams LT. Structural and functional diversity in the FGF receptor multigene family. Adv Cancer Res 1993 ; 60 : $1-41$.

6. Amalric F, Bouche G, Bonnet H, Brethenou P, Roman AM, Truchet I, Quarto N. Fibroblast growth factor-2 (FGF-2) in the nucleus: translocation process and targets. Biochem Pharmacol 1994 ; 47 : 111-5.

7. Burrus LW, Zuber ME, Lueddecke BA, Olwin BB. Identification of a cysteine-rich receptor for fibroblast growth factors. $\mathrm{Mol}$ Cell Biol 1992 ; 12 : 5600-9.

8. Steegmaler M, Levinovitz A, Isenmann S, Borges E, Lenter M, Kocher HP, Kleuser B, Vestweber D. The E-selectin-ligand ESL-1 is a variant of a receptor for fibroblast growth factor. Nature 1995 ; 373 : 615-20.

9. Coulier F, Pizette S, Batoz M, Birnbaum D. Les récepteurs des facteurs de croissance des fibroblastes: une famille qui s'agrandit. médecine/sciences $1992 ; 8$ : 811-8.

10. Werner S, Duan DSR, De Vries C, Peters KG, Johnson DE, Williams LT. Differential splicing in the extracellular region of fibroblast growth factor receptor 1 generates receptor variants with different ligand-binding specificities. Mol Cell Biol 1992; 12: 82-8

11. Chellaiah AT, McEwen DG, Werner S, $\mathrm{Xu}$ J, Ornitz DM. Fibroblast growth factor receptor (FGFR)3. Alternative splicing in immunoglobulin-like domain 3 creates a receptor highly specific for acidic FGF/FGF-1. raît varier dans certains tissus tumoraux. On a montré, en effet, que les cellules du pancréas humain, normal ou cancéreux, présentent les formes de FGFR $_{1}$ qui possèdent deux structures Ig, deux Ig plus une insertion ou trois Ig, mais que l'isoforme à deux Ig est surexprimée dans l'adénome pancréatique [13].

La combinaison des modifications potentielles prédécrites peut théoriquement déterminer l'activité de chacun des variants ainsi formé. Quoique la fonction des séquences altérées soit loin d'être complètement élucidée, on peut en effet proposer un rôle régulateur pour un certain nombre d'entre elles [5, 1418]: (1) les structures Ig-2 et Ig-3 sont nécessaires à la liaison du ligand ; Ig-1 ne l'est pas mais sa présence paraît abaisser l'affinité des récepteurs. Cela suggère que Ig-1 interagit avec Ig-2 et Ig-3 ou avec le site de liaison. (2) La «boîte" acide joue vraisemblablement un rôle dans la stabilisation de la conformation du site de liaison, quoique son absence n'affecte apparemment pas l'affinité de $\mathrm{FGFR}_{1}$ et de $\mathrm{FGFR}_{2}$ pour leurs ligands. (3) Il semble que la phosphorylation du dipeptide TV par une PKC puisse assurer la rétrorégulation négative des FGFR; ce phénomène a été récemment dé-

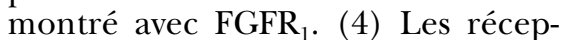
teurs FGFR $_{1(\mathrm{~b})}, \mathrm{FGFR}_{2(\mathrm{~b})}$ et $\mathrm{FGFR}_{3(\mathrm{~b})}$ ont une affinité beaucoup plus importante pour $\mathrm{FGF}_{1}$ et $\mathrm{FGF}_{7}$ que pour $\mathrm{FGF}_{2}$; en revanche, les $\mathrm{FGFR}_{1(\mathrm{c})}, \mathrm{FGFR}_{2(\mathrm{c})}$ et $\mathrm{FGFR}_{3(\mathrm{c})}$ ont une affinité pratiquement identique pour $\mathrm{FGF}_{1}$ et $\mathrm{FGF}_{2}$ mais ne lient pas $\mathrm{FGF}_{7}$. Le récepteur $\mathrm{FGFR}_{4}$, qui possède la séquence $c$, a une affinité plus forte pour $\mathrm{FGF}_{1}$ que pour $\mathrm{FGF}_{2}$ et $\mathrm{FGF}_{7}$. Curieusement, les variants solubles ayant une séquence épissée a sont les seules formes caractérisées à avoir une affinité plus forte pour $\mathrm{FGF}_{2}$ que pour $\mathrm{FGF}_{1}$. Il apparaît que la fixation de $\mathrm{FGF}_{1}$ et $\mathrm{FGF}_{2}$ implique respectivement Ig-2 et Ig-3, alors que l'affinité optimale pour $\mathrm{FGF}_{7}$ nécessite l'implication à la fois de Ig-2 et de Ig-3. Signalons aussi que FGFR ${ }_{2(c)}$ présente une affinité beaucoup plus importante pour $\mathrm{FGF}_{3}$ que $\mathrm{FGFR}_{1(\mathrm{c})}$, $\mathrm{FGFR}_{3(\mathrm{c})}$ et $\mathrm{FGFR}_{4}$. (5) Les régions intracytoplasmiques de type $\mathrm{t}$ sont dépourvues d'activité tyrosine kinase ; en revanche, les particularités de fonctionnement du type s (comparé au type non tronqué $r$ ) ne sont pas connues. (6) L'autophosphorylation des tyrosines du domaine intracellulaire n'a été prouvée que dans quelques cas $\left(\mathrm{Y}_{653}\right.$ et $\mathrm{Y}_{766}$ pour $\mathrm{FGFR}_{1}$, $\mathrm{Y}_{655}$ et $\mathrm{Y}_{768}$ pour $\mathrm{FGFR}_{2}$, par exemple) (Tableau II) ; ce processus est impliqué dans le contrôle de la fixation et de l'activation de certains effecteurs.

\section{La transmission membranaire des signaux: un mécanisme complexe d'interface}

L'activité des FGF et de leurs récepteurs est réglée par au moins deux catégories de composés du milieu péricellulaire. D'une part, on a abondamment montré que la présence des HSPG ou de l'héparine est nécessaire pour que les FGF puissent agir efficacement sur la plupart des lignées cellulaires en culture [4]. Il apparaît que les HSPG ont une grande capacité de fixation des FGF mais les lient généralement avec une affinité beaucoup plus faible que leurs récepteurs. En outre, ils s'associent spécifiquement à une séquence amino-terminale de Ig-2 des FGFR. On considère communément que ces composés sont impliqués dans la réponse biologique aux FGF, bien que leur rôle exact en tant que cofacteurs soit encore très discuté [4]. Les HSPG associés à la matrice extracellulaire paraissent avoir une fonction de stockage pour les FGF exogènes ; un mécanisme protéolytique pourrait assurer leur libération sur demande. En revanche, les HSPG associés à la membrane plasmique ont plutôt la propriété de permettre ou de stimuler l'activation des FGFR par leurs ligands, jouant ainsi un rôle de corécepteurs (figure 2). Le mécanisme intime qui assure ce rôle n'est pas encore élucidé de façon très claire. En particulier, il n'est pas aisé de savoir si les HSPG membranaires servent à stabiliser les interactions entre les facteurs et leurs récepteurs, ou si ces glycanes accroissent la liaison des facteurs ou l'activation des récepteurs via des changements conformationnels des uns et/ou des autres. D'autre part, des travaux prouvent qu'une séquence détenue par plusieurs types de molécules d'adhérence cellulaire (CAM) est isologue d'un 


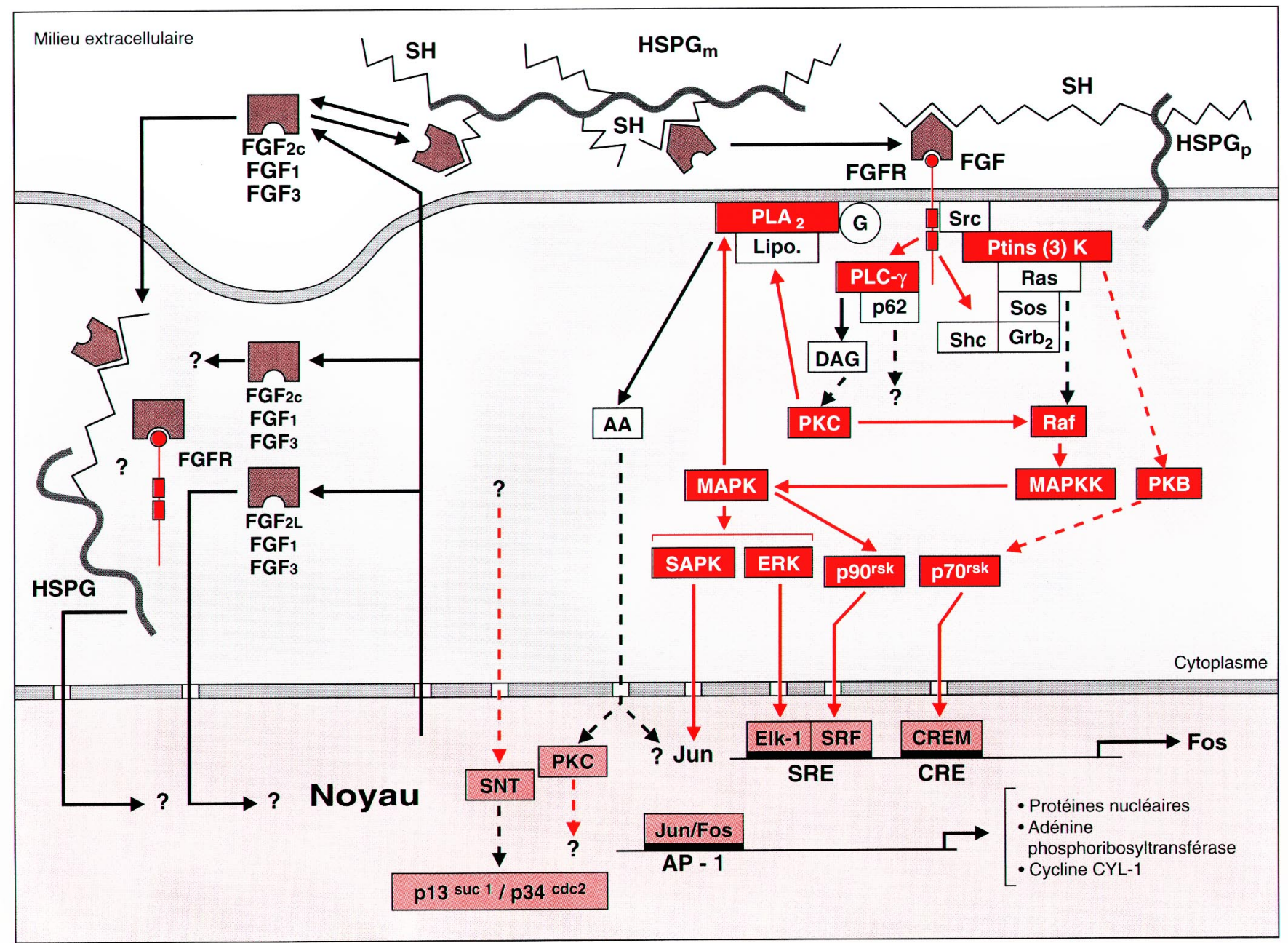

Figure 2. Voies de signalisation des FGF. On admet que les FGF peuvent activer deux voies de signalisation: une voie dans laquelle ils agissent directement sur des cibles nucléaires; une voie indirecte qui comprend un système de transduction membranaire et des cascades de protéines transductionnelles et de messagers. (1) Activation directe. Les formes traduites des FGF ont trois destinées possibles: certaines formes sont sécrétées; d'autres restent dans le cytoplasme et agissent sur des cibles inconnues et/ou sont dégradées; d'autres encore sont directement transférées dans le noyau sans dégradation préalable. Ce phénomène a été démontré pour FGF $F_{1}$ pour la forme courte et les trois formes longues de $\mathrm{FGF}_{2}\left(\mathrm{FGF}_{2 C}\right.$ et $\left.\mathrm{FGF}_{2 L}\right)$ et pour $\mathrm{FGF}_{3}$. Les formes extracellulaires sont, soit stockées par les $\mathrm{HSPG}_{\mathrm{m}}$ soit internalisées, probablement via des FGFR et des HSPG, et transportées dans le noyau. Seules, quelques cibles de la forme $F_{G C}$ ont été jusqu'à présent caractérisées. (2) Activation indirecte. Les HSPG facilitent ou augmentent la capacité des FGF (relargués par les HSPG en fonction de la demande) de stimuler les FGFR. L'une des hypothèses envisagées actuellement admet que les HSPG pourraient agir en modifiant la conformation du FGF ( 2 : FGF non fixé aux HSPG; : FGF fixé aux HSPG). Cette activation déclenche la stimulation d'au moins trois effecteurs (PLC $\gamma$, p60 src, protéine Shc), qui peut entraîner l'activation d'une ou plusieurs cascades interdépendantes de protéines de transmission du signal et de messagers secondaires, suivie de l'induction des gènes précoces (en particulier de FOS, JUN et MYC) et de nombreux gènes tardifs (en particulier du gène codant pour la cycline CYL1, impliquée dans le contrôle de la transition $G_{1} / S$ du cycle cellulaire). Dans certains systèmes neuronaux (cas des cellules PC12), ce processus aboutit également à la phosphorylation d'une protéine appelée SNT. Cette protéine, en agissant sur le complexe $p 13^{\text {suc-1 }} /$ p34 $^{\text {cdc2 }}$, pourrait être impliquée dans l'inhibition de la mitose qui précède l'induction de la différenciation. Les flèches noires pleines représentent une production et/ou un déplacement de composés; les flèches noires discontinues représentent une activation par un mécanisme indéterminé ; les flèches rouges pleines représentent une phosphorylation directe; les flèches rouges discontinues représentent une phosphorylation par un mécanisme indéterminé. CREM: facteurs de transcription de la famille CREB, activés par l'AMPc;CRE : cyclic AMP response element; SRF: serum response factor ; Elk1 : facteur de transcription de la famille Ets formant un complexe avec SRF fixé sur son site ; HSPG ${ }_{m}$ : protéoglycanes à sulfate d'héparane de la matrice extracellulaire ; $H S P G_{p}$; protéoglycanes à sulfate d'héparane membranaires ; SH : sulfate d'héparane; PLA $A_{2}$ : phospholipase $A_{2} ; G$ : protéine $G ;$ Src: tyrosine kinase, produit du proto-oncogène p60 5 irc PtIns (3) K: phosphatidyl inositol 3 kinase ; Lipo: lipocortine ; PLC: phospholipase C; DAG: diacylglycérol ; Shc: adaptateur à domaine $S H 2$; Sos : son of sevenless, protéine d'échange: Grb ${ }^{2}$ : adaptateur à domaine $S H 2$ et $S H 3$; PKB: protéine kinase $B$; $A A$ : acide arachidonique ; MAPK: mitogen activated protein kinase ; MAPKK : MAPK kinase ; SAPK : stress-activated protein kinase ; ERK: extracellular regulated protein kinase; $p 90^{\text {rsk }}$ et $p 70^{\text {rsk }}$ : sérine thréonine kinases qui activent la protéine ribosomique S6; SNT: suc associated neurotrophic factor-induced tyrosine-phosphorylated target. 


\section{RÉFÉRENCES}

12. Werner S, Weinberg W, Liao X, Peters $\mathrm{KG}$, Blessing M, Yuspa SH, Weiner RL, Williams LT. Targeted expression of a dominant-negative FGF receptor mutant in the epidermis of transgenic mice reveals a role of FGF in keratinocyte organization and differentiation. EMBO J 1993; 12 : 263543.

13. Kobrin MS, Yamanaka Y, Friess H, Lopez ME, Korc M. Aberrant expression of type 1 fibroblast growth factor receptor in human pancreatic adenocarcinomas. Cancer Res 1993 ; 53 : 4741-4.

14. Hou J, McKeehan K, Kan M, Carr SA, Huddleston MJ, Crabb JW, McKeehan WL. Identification of tyrosines 154 and 307 in the extracellular domain and 653 and 766 in the intracellular domain as phosphorylation sites in the heparin-binding fibroblast growth factor receptor tyrosine kinase (flg). Protein Sci 1993 ; 2 : 86-92.

15. Cheon HG, LaRochelle WJ, Bottaro DP, Burgess WH, Aaronson SA. High-affinity binding sites for related fibroblast growth factor ligands reside within different receptor immunoglobulin-like domains. Proc Natl Acad Sci USA 1994; 91 : 989-93.

16. Zimmer Y, Givol D, Yayon A. Multiple structural elements determine ligand binding of fibroblast growth factor receptors. $J$ Biol Chem 1993 ; 268 : 7899-903.

17. Mathieu M, Kiefer P, Mason I, Dickson C. Fibroblast growth factor (FGF) 3 from $X e-$ nopus laevis (XFGF3) binds with high affinity to FGF receptor 2. J Biol Chem $1995 ; 270$ : 6779-87.

18. Gillespie LL, Chen G, Paterno GD. Cloning of a fibroblast growth factor receptor 1 splice variant from Xenopus embryos that lacks a protein kinase $\mathrm{C}$ site important for the regulation of receptor activity. $J$ Biol Chem 1995 ; 270 : 22758-63.

19. Williams EJ, Furness J, Walsh FS, Doherty P. Activation of the FGF receptor underlies neurite outgrowth stimulated by L1, NCAM, and N-Cadherin. Neuron 1994; 13 : 583-94.

20. Van der Geer P, Hunter T. Receptor protein-tyrosine kinases and their signal transduction pathways. Annu Rev Cell Biol $1994 ; 10$ : 251-337.

21. Zhan X, Plourde C, Hu X, Friesel R, Maciag T. Association of fibroblast growth factor receptor-1 with c-src correlates with association between c-src and cortactin. I Biol domaine présent dans les FGFR, dans la région comprise entre Ig-1 et Ig-2 [19]. Ce domaine est impliqué dans la régulation de la plasticité neuritique des neurones cérébelleux contrôlée par les CAM, suggérant que ces dernières pourraient moduler directement ou indirectement le fonctionnement des FGFR activés par les FGF.

Il est maintenant acquis que la fixation des FGF sur les monomères de FGFR déclenche la dimérisation de ces derniers [5]. Le mécanisme qui induit le processus de dimérisation des FGFR n'est pas connu avec certitude, mais il semble que les HSPG pourraient être impliqués dans son déclenchement. Des auteurs, en effet, ont proposé un modèle dans lequel l'équilibre entre les molécules de ligand libre et celles de ligand lié est coopérativement déplacé dans le sens de la liaison quand un complexe se forme entre le récepteur, les HSPG et le ligand [4]. La dimérisation des FGFR, à l'instar des autres récepteurs à activité tyrosine kinase, stimule cette activité des récepteurs et induit ainsi l'autophosphorylation intra-moléculaire et/ou inter-moléculaire de plusieurs de leurs tyrosines (Tyr). Ce processus déclenche l'association aux récepteurs et/ou la phosphorylation directe (sur des Tyr) de plusieurs protéines qui jouent le rôle d'effecteurs [20].

On a montré jusqu'à présent que les FGFR peuvent contracter une association directe avec au moins deux effecteurs enzymatiques : la PLC- $\gamma$ et la $\mathrm{p} 60^{\mathrm{src}}$; elle est réalisée par l'ancrage des domaines de type $\mathrm{SH}_{2}$ (src homology 2) des deux enzymes avec les sites autophosphorylés des FGFR $[20,21]$. Des travaux réalisés in vitro prouvent que cette association conduit à une stimulation importante de l'activité des deux enzymes ; dans le cas de la PLC- $\gamma$, d'ailleurs, la phosphorylation directe de cette dernière par les récepteurs est une étape intermédiaire obligatoire. Gageons que d'autres associations sont encore à découvrir. Par exemple, un tel mécanisme est théoriquement possible avec la SH-PTP2, une tyrosine phosphatase qui pourrait être notamment impliquée dans l'activation de la p60 $0^{\text {sc }}[20,22]$. En outre, un certain nombre de substrats paraissent être directement phospho- rylés par les récepteurs des FGF; l'un d'entre eux est actuellement bien caractérisé : il s'agit de l'adaptateur Shc [23] (figure 2). Récemment, Shi et al. ont tenté d'élucider le rôle que pourrait jouer la nature des variants dimérisés de FGFR $_{1}$ dans l'accrochage et l'activation de la PLC- $\gamma$ [24]. Ils ont ainsi montré que les couples homodimériques actifs sont les seuls à pouvoir à la fois accrocher et activer la PLC- $\gamma$ via respectivement l'autophosphorylation intramoléculaire de Tyr 766 et l'autophosphorylation trans-moléculaire de Tyr 653; les couples hétérodimériques formés d'un variant actif et d'un variant inactif ne peuvent, quant à eux, qu'accrocher l'effecteur sur la Tyr 766 phosphorylée et non l'activer. Ce travail très élégant suggère que l'activité des effecteurs associés aux récepteurs peut être contrôlée par la combinaison des variants qui se dimérisent. Il est donc proposé, pour la première fois, un mécanisme de transduction membranaire qui intègre le fait qu'il existe généralement plusieurs isoformes de récepteurs dans chaque type cellulaire.

\section{La transmission intracellulaire des signaux: un réseau à multiples relais}

Les FGFR, via les effecteurs précédemment décrits, possèdent la propriété d'activer de nombreuses protéines de la cascade de transmission du signal (Sos, Ras, PKC, Raf, MAPKK*, MAPK, PtIns(3)K, PLA 2 , lipocortines, protéine kinase $\mathrm{B}$ ( $\mathrm{PKB})$, $\left.\mathrm{p} 70^{R S K}, \mathrm{p} 90^{R S K}\right)$ et des messagers secondaires (acide arachidonique, inositol phosphates, diacylglycérol [DAG], calcium) $[17,20,23,25-31]$ (figure 2 ); d'autres restent certainement à découvrir. Ces protéines dialoguent principalement par des interactions moléculaires et des réactions de phosphorylation. Celles-ci ne sont pas spécifiques des FGF, puisqu'elles sont impliquées dans la transmission du signal de nombreux facteurs de croissance et neurotrophines [20]; mais le réseau de relais qu'elles forment présente des particularités propres aux

\footnotetext{
$\overline{* \text { Voir glossaire, }}$ p. 312.
} 


\section{RÉFÉRENCES}

22. Tang TL, Freeman RM, O'Reilly AM, Neel BG, Sokol SY. The SH2-containing protein-tyrosine phosphatase SH-PTP2 is required upstream of MAP kinase for early $\mathrm{X} \boldsymbol{e}$ nopus development. Cell 1995 ; 80 : 473-83.

23. Vainikka S, Joukov V, Wennström S, Bergman M, Pelicci PG, Alitalo K. Signal transduction by fibroblast growth factor receptor-4 (FGF-4). J Biol Chem 1994 ; 269 : 18320-6.

24. Shi E, Kan M, Xu J, Wang F, Hou J, McKeehan WL. Control of fibroblast growth factor receptor kinase signal transduction by heterodimerization of combinatorial splice variants. Mol Cell Biol 1993 ; 13 : 3907-18.

25. Huang J, Mohammadi M, Rodrigues GA, Schlessinger J. Reduced activation of RAF-1 and MAP kinase by a fibroblast growth factor receptor mutant deficient in stimulation of phosphatidylinositol hydrolysis. J Biol Chem 1995 ; 270 : 5065-72.

26. Burgering BMTh, Coffer PJ. Protein kinase B (c-Akt) in phosphatidylinositol-3-OH kinase signal transduction. Nature 1995; $376: 5-7$.

27. Marshall CJ. Specificity of receptor tyrosine kinase signalling: transient versus sustained extracellular signal-regulated kinase activation. Cell $1995 ; 80: 179-85$.

28. L'Allemain G. Deciphering the MAP kinase pathway. Prog Growth Factor Res 1994 ; 5 : 291-334.

29. Patte C, Rothhut B, Russo-Marie F, Blanquet PR. Possible involvement of a lipocortin in the initiation of DNA synthesis by human endothelial cells. Exp Cell Res 1991; $197: 12-20$

30. Sa G, Murugesan G, Jaye M, Ivashchenko Y, Fox PL. Activation of cytosolic phospholipase A2 by basic fibroblast growth factor via a $\mathrm{p} 42$ mitogen-activated protein kinase-dependent phosphorylation pathway in endothelial cells. J Biol Chem 1995 ; 270 : 2360-6.

31. Patte C, Blanquet PR. Possible involvement of arachidonic acid metabolites in the synergistic action of endothelial mitogenesis by basic fibroblast growth factor and phorbol ester. Cell Mol Biol 1992 ; 38 : 429-36.

32. Rabin SJ, Cleghon V, Kaplan DR. SNT, a differentiation-specific target of neurotrophic factor-induced tyrosine kinase activity in neurons and PC12 cells. Mol Cell Biol $1993 ; 13: 2203-13$.

33. Coffer PL, Woodgett JR. Differential subcellular localisation of two isoforms of p70 S6 protein kinase. Biochem Biophys Res Commun 1994 ; 198 : 780-6.

$m / s n^{\circ} 3$, vol. 12 , mars 96
FGF. Par exemple, la stimulation successive du complexe Shc-Grb $\mathrm{S}_{2}-\mathrm{Sos}$, de Ras et des sérine/thréonine kinases Raf, MAPKK et MAPK est apparemment induite par la phosphorylation de Shc et non par l'association directe de Shc aux récepteurs du FGF. Ces derniers, en effet, ne possèdent pas le site spécifique putatif de liaison pour le domaine $\mathrm{SH}_{2}$ de Shc. En revanche, Shc et $\mathrm{Grb}_{2}$ peuvent s'associer respectivement au récepteur du NGF (nerve growth factor) et à celui de l'EGF (epidermal growth factor). Précisons, à propos de cette cascade de signaux, que la MAPK activée par le FGF phosphoryle de nombreux substrats cytoplasmiques parmi lesquels il faut citer la p90 ${ }^{R S K}$. Autre exemple: la PtIns $(3) \mathrm{K}$ est stimulée par les récepteurs FGFR $_{1}$ et $\mathrm{FGFR}_{4}$ du FGF sans s'associer à ces récepteurs via sa région $\mathrm{SH}_{2}$, contrairement à ce qui est observé avec d'autres récepteurs de facteurs de croissance. Des travaux récents montrent que cette enzyme, sous l'impulsion de $\mathrm{FGF}_{2}$, déclenche la phosphorylation et la stimulation de la p70 via la phosphorylation et l'activation d'une nouvelle kinase appelée PKB. Les relais en amont qui permettent aux FGFR d'activer la PtIns(3)K sont encore inconnus, quoique des études récentes suggèrent que l'activité de l'enzyme pourrait être contrôlée par une association directe de celle-ci à Ras et à la p60 $60^{s r}$.

Le FGF, probablement par une voie qui implique la p60sc, est capable d'induire la phosphorylation d'une protéine appelée SNT (suc-associated neurotrophic factor-induced tyrosine-phosphorylated target), qui s'associe dans le noyau au complexe p13 $13^{S U C-1} / 34^{C D C 2}$ impliqué dans le contrôle de la mitose [32]. En outre, certaines sérine/ thréonine kinases peuvent migrer par translocation dans le noyau (figure 2). Ce phénomène a été démontré pour plusieurs isoformes de la MAPK $\left(\mathrm{ERK}_{1}, \mathrm{ERK}_{2}, \mathrm{SAPK}\right)$, pour l'une des deux isoformes connues de la $\mathrm{p} 70^{R S K}$ et pour la p90 ${ }^{R S K}[20,28,33,34]$. On considère actuellement que les ERK activent la séquence consensus SRE (serum response element) du promoteur des gènes précoces; dans le cas du gène $F O S$, cette activation est obtenue par la phosphorylation des régulateurs transcriptionnels Elk-1 et SAP1. Un travail récent a également montré que les SAPK transloquées phosphorylent et activent le gène $\int U N\left(m / s n^{\circ}\right.$, vol. 11, p. 467) [34]. Rappelons que l'induction de l'expression des gènes précoces FOS, $J U N$ et $M Y C$ est l'un des résultats les plus habituels de l'action des FGF. De nombreux gènes tardifs sont également stimulés par les FGF. Par exemple, le $\mathrm{FGF}_{2}$ active les gènes codant pour des protéines nucléaires associées à l'ADN, pour l'adénine phosphoribosyltransférase et pour la cycline CYL1, vraisemblablement impliquées dans le contrôle de la prolifération [35]. Le rôle des gènes précoces dans le mécanisme qui aboutit à ces processus transcriptionnels est indéterminé. D'autre part, $\mathrm{p} 70^{R S K}$ et p90 ${ }^{R S K}$ sont théoriquement capables de stimuler les séquences consensus CRE (cyclic AMP response element) et SRE présentes dans le promoteur de nombreux gènes (en particulier les gènes précoces) via la phosphorylation des régulateurs transcriptionnels CREM et SRF [36, 37]. Précisons aussi que l'acide arachidonique possède la propriété d'activer certaines isoformes de PKC ainsi que la séquence HSE du promoteur du gène des protéines heat shock HSP70 et HSP90, dont on commence à découvrir l'importance pour le fonctionnement des processus de signalisation [38, 39]. Cependant, l'implication directe de ces deux mécanismes dans la signalisation des FGF n'a pas encore été recherchée.

Les FGF ont aussi la curieuse propriété de pouvoir pénétrer dans le milieu nucléaire sans dégradation préalable. Ainsi, quatre formes traduites de $\mathrm{FGF}_{2}$ ont été caractérisées : une forme courte, dont la transcription commence à un codon AUG, et trois formes longues, dont la transcription commence à un codon CUG. La forme courte demeure essentiellement cytoplasmique et peut être externalisée pour activer le système de transduction membranaire de la cellule sécrétrice (action autocrine) ou d'une autre cellule proche (action paracrine) ou éloignée (action exocrine). En revanche, les formes longues sont transférées dans le noyau et pourraient n'être relarguées dans le milieu extracellulaire que lorsqu'il y a destruction et mort cellulaire. Cependant, les deux catégories de formes, quand elles sont externalisées, peuvent être partielle- 
ment transférées dans le noyau, une partie d'entre elles étant dégradée par protéolyse [6, 40] (figure 2). Curieusement d'ailleurs, la forme sécrétée peut stimuler la synthèse des formes endogènes [41]. La forme courte internalisée s'accumule dans le nucléole, contrairement aux formes longues présentes dans le noyau. Dans le cas de l'endothélium aortique bovin, par exemple, on a montré que la forme courte internalisée entre dans le noyau durant la transition $G_{o} / G_{1}$ du cycle cellulaire et stimule l'expression de la nucléoline et sa phosphorylation (vraisemblablement par une caséine-kinase 2) ainsi que la transcription des gènes ribosomiques [6]. Le $\mathrm{FGF}_{1}$ et le $\mathrm{FGF}_{3}$ oncogéniques peuvent être également transportés dans le noyau $[42,43]$ (figure 2). Le mécanisme qui contrôle le transport nucléaire a fait l'objet de nombreuses investigations mais reste encore très discuté. La translocation des formes endogènes paraît être réglée par une séquence de type NLS (nuclear localization sequence), alors que l'internalisation des formes exogènes pourrait plutôt dépendre des récepteurs et/ou des HSPG. En particulier, d'intéressants travaux réalisés par Amalric et al. (Toulouse, France) ont montré que la forme courte sécrétée de $\mathrm{FGF}_{2}$ peut être directement transportée dans le noyau par des HSPG [6]. L'urgence est maintenant de caractériser les cibles intracellulaires de ces facteurs et d'étudier le rôle exact des récepteurs et des HSPG dans leur trafic intracellulaire. Les seules données disponibles sont celles qui ont été publiées par le groupe précité [6]; elles montrent que la forme courte de $\mathrm{FGF}_{2}$ présente dans le noyau possède plusieurs cibles directes (domaine en amont du site d'initiation des gènes ribosomiques ; histones $\mathrm{H} 1$; caséine kinase 2) qui sont impliquées dans l'activation transcriptionnelle des gènes qui codent pour l'ARN ribosomique.

43. Kiefer P, Dickson C. Nucleolar association of fibroblast growth factor 3 via specific sequence motifs has inhibitory effects on cell growth. Mol Cell Biol 1995 ; 15 : 4364-74.

44. Wanaka A, Johnson EM, Milbrandt J. Localization of FGF receptor mRNA in the adult rat central nervous system by in situ hybriditation. Neuron $1990 ; 5$ : 267-81.

45. Torriglia A, Blanquet PR. Immunochemical evidence for a fibroblast growth factor receptor in adult retinal optic fiber and sy-
Quatre groupes de travaux indiquent que la plupart des relais sont dotés d'une extraordinaire plasticité qui leur permet de contrôler de nombreuses fonctions :

- La MAPK joue un rôle essentiel dans le contrôle de la mitogenèse exercée par les FGF. Son rôle paraît également crucial pour l'induction de la différenciation du modèle en culture PC12 (cellules issues de phéochromocytome de rat) par ce facteur $[17,20,23,25,27]$. Les facteurs de croissance qui déclenchent la différenciation des cellules PC12 (FGF, NGF, PDGF) induisent à la fois une stimulation soutenue et une translocation nucléaire de la MAPK, alors que les facteurs mitogènes pour ces cellules (EGF, $\mathrm{IGF}_{1}$ ) n'activent la kinase que transitoirement [27]. Bien que ce processus demeure encore énigmatique sur bien des points, cela suggère que les récepteurs des FGF pourraient sélectionner des modes d'activation différents de la MAPK pour déclencher, soit le cycle de prolifération, soit la différenciation neuronale. Remarquons, cependant, qu'aucune donnée n'a été jusqu'à présent obtenue concernant la transmission du signal FGF dans des systèmes neuronaux autres que les PC12, en dépit de l'effet neurotrophique exercé par ces facteurs et de la localisation de leurs récepteurs dans de nombreuses régions du système nerveux central $[44,45]$.

- L'acide arachidonique produit grâce à l'action de la PLA PLtoplas- $_{2}$ mique joue un rôle important dans le contrôle de la prolifération des cellules endothéliales vasculaires par le $\mathrm{FGF}_{2}$ [29-31]. Quoique le mécanisme de l'activation de la $\mathrm{PLA}_{2}$ soit encore un sujet de controverse, il semble qu'elle soit activée via sa phosphorylation par une PKC ou la MAPK, ou via la phosphorylation d'une protéine de type annexine/lipocortine, selon l'origine des cellules et la fonction concernée [29, 30,46]. Mais le $\mathrm{FGF}_{2}$ peut aussi contrôler la migration des cellules endothéliales vasculaires en stimulant une PLA P $_{2}$ cytoplasmique et la formation d'acide arachidonique via l'activation d'une protéine G [47].

- L'association de la PLC- $\gamma$ au FGFR et l'activation qui en résulte (l'hydrolyse par l'enzyme du DAG (diacylglycérol) et des inositol-phosphates suivie de la libération du calcium intracellulaire) n'est pas nécessaire 
pour que le FGF déclenche la prolifération cellulaire. En revanche, ce processus serait important pour l'internalisation du récepteur [48]. En fait, il apparaît maintenant que FGFR $_{1}$ peut stimuler la MAPK et contrôler la prolifération en activant les deux voies parallèles de Ras et de la PLC- $\gamma$ [25]. Dans ce mécanisme, la PLC- $\gamma$ paraît agir sur Raf via l'activation d'une PKC (figure 2). Remarquons cependant qu'une protéine récemment découverte, appelée p62, contenant cinq sites de liaison pour le domaine $\mathrm{SH}_{3}$ et de multiples sites phosphorylés spécifiques du domaine $\mathrm{SH}_{2}$, possède la curieuse propriété de pouvoir complexer la PLC- $\gamma$ avec des protéines aussi diverses que la $\mathrm{Grb}_{2}$, la p60 $0^{\text {sc }}$ et la GAP (GTPase activating protein) [49]. Il est donc tentant de penser que d'autres relais en aval, jusqu'ici insoupçonnés, pourraient aussi permettre à la PLC- $\gamma$ d'activer la MAPK. Le fait que la prolifération puisse être déclenchée par le domaine $\mathrm{SH}_{3}$ d'une PLC- $\gamma$ microinjectée est tout à fait en faveur de cette hypothèse [50]. Par conséquent, l'activation de la prolifération par la MAPK ne nécessite pas obligatoirement une stimulation de la PLC$\gamma$, mais elle semble néanmoins pouvoir être déclenchée par plusieurs voies alternatives ou additionnelles contrôlées par cette enzyme. Ajoutons, en outre, que la PLC- $\gamma$ peut être impliquée dans le contrôle de la plasticité neuritique exercée par le FGF et les CAM, via la libération de DAG suivie de la production d'acide arachidonique par une lipase ; cela a été observé dans des neurones cérebelleux [51].

- Les expérimentations précédemment relatées suggèrent fortement que les facteurs $\mathrm{FGF}_{1}$ et $\mathrm{FGF}_{2}$ intranucléaires pourraient être capables de déclencher directement la synthèse de l'ADN et la transition $\mathrm{G}_{1} / \mathrm{S}$ du cycle cellulaire. Cependant, dans certains cas, il semble que ce processus doive être nécessairement coordonné à un mécanisme plus classique de signalisation, impliquant des récepteurs à tyrosine kinase, pour que la synthèse d'ADN aboutisse effectivement à la mitogenèse [52]. En revanche, on a récemment montré que la forme nucléaire de $\mathrm{FGF}_{3}$ inhibe la synthèse d'ADN et la mitogenèse [43]. Quelques travaux montrent aussi que la synthèse intracrine de $\mathrm{FGF}_{1}$ et $\mathrm{FGF}_{2}$ peut participer au contrôle de certains états différenciés [41, 53]. Bien que ces phénomènes soient encore très mal compris, il est possible, selon les types cellulaires ou les stades du développement, que ce système de régulation directe puisse remplacer, court-circuiter ou compléter de façon coordonnée certaines cascades classiques de transmission du signal.

\section{Conclusion et futures directions}

L'étude de la signalisation des FGF dans les tissus normaux et pathologiques a abouti à un extraordinaire patchwork de données qui est encore loin d'être complètement compréhensible. En particulier, la plupart des travaux ont été réalisés avec $\mathrm{FGF}_{1}$ et $\mathrm{FGF}_{2}$; peu ont impliqué les autres membres de la famille des FGF. En outre, de très nombreux points restent à éclaircir ou à élucider pour établir une cartographie complète des relais qui assurent la transmission des signaux de la membrane plasmique jusqu'au noyau. Cependant, d'ores et déjà, l'explosion des connaissances de ces dernières années permet de conclure que les multiples fonctions des FGF sont vraisemblablement contrôlées par une combinatoire de relais transductionnels. Pour rechercher la mécanique intime qui anime cette combinatoire, il faut maintenant étudier le rôle des différents types de récepteurs et de leurs principales isoformes dans la sélection des cascades de relais. Quelques expérimentations ont abordé ce thème. Des travaux récents, par exemple, ont montré que FGFR $_{1}$ phosphoryle beaucoup plus fortement l'adaptateur Shc, la PLC- $\gamma$, Raf et les isoformes $\mathrm{ERK}_{1}$ et $\mathrm{ERK}_{2}$ que $\mathrm{FGFR}_{4}$ [23]. Mais ces approches restent encore trop rares et trop sommaires. L'étude de cette mécanique nécessite également la caractérisation des éléments moléculaires qui déterminent chaque protéine impliquée dans la cascade de transmission des signaux à entretenir un type de relais plutôt qu'un autre. Rappelons que chaque protéine de la transmission du signal, dans son appellation, n'est en réalité que la forme générique 
d'une famille d'isoformes qui grandit sans cesse ; c'est le cas, par exemple, des MAPK, MAPKK, Raf et Ras [20, 28]. Chaque isoforme paraît avoir une spécificité d'action et de localisation intracellulaire. Ces dernières années, des travaux réalisés dans des domaines très divers ont abouti à l'émergence d'une nouvelle génération de protéines qui paraissent dotées d'intéressantes propriétés interrelationnelles. Par exemple, les RACK (receptors for activated C-kinase) et la protéine 14-3-3 s'associent respectivement à certaines isoformes de la PKC et à Raf-1 [54]. De même, la stathmine peut être phosphorylée par de nombreuses sérine/thréonine kinases (en particulier par la MAPK) et peut potentiellement interagir avec une grande variété de cibles [55]. Beaucoup d'informations conduisent à penser que ces protéines pourraient servir à sélectionner des isoformes de ces protéines de transmission du signal et à diriger leur activité vers telle ou telle cible. Il est donc tentant de spéculer que ces «intégrateurs » pourraient régler la combinaison des relais nécessaires à chaque fonction des FGF. Enfin, la recherche des processus de régulation contrôlés par l'accumulation intranucléaire des FGF doit aussi être abordée en urgence. En particulier, il est maintenant nécessaire de rechercher et d'étudier les points de jonction qui pourraient exister entre ces processus et les cascades de protéines qui transmettent le signal. Il ne serait pas étonnant que la coordination de ces deux voies puisse assurer une spécificité de réponse aux FGF dans certains systèmes cellulaires
* GLOSSAIRE *

CRE : cAMP - responsive element.

CREM : $c A M P$ - responsive element modulator.

$D A G$ : diacylglycérol.

EGF : epidermal growth factor.

Elk-1 : ets 1 - like gene.

ERK : extracellularly regulated protein kinase.

GAP : GTPase - activating protein.

Grb2 : growth factor receptor - bound protein 2.

HSE : heat shock protein response element.

HSPG : heparin sulfate proteoglycans

IGF : insulin-like growth factor.

MAPK : mitogen - activated protein kinase ou ERK.

MAPKK : MAPK kinase ou MEK (mitogenically extraregulated kinase).

NGF : nerve growth factor.

$P D G F$ : platelet-derived growth factor.

$\boldsymbol{P K C}$ : protéine kinase $\mathrm{C}$.

PLA2 : phospholipase $\mathrm{A}_{2}$.

$P L C-\gamma$ : phospholipase C- $\gamma$.

PtIns(3)k : phosphatidylinositol 3kinase.

RSK : ribosomal S6 kinase.

SAPK : stress - activated protein kinase.

Shc : SH2 containing protein.

SH-PTP : SH2 domain - containing protein - tyrosine phosphatase.

Sos : son of sevenless.

SRE : serum response element.

SRF : serum response factor.

\section{TIRÉS À PART}

P.R. Blanquet.

\section{Summary}

FGF signalling: a mechanism on the way of being deciphered

Fibroblast growth factors (FGF) are encoded by at least nine distinct genes. They share a broad spectrum of biological functions and appear to be involved in various physiopathological processes including tumor development, atherogenesis, neurodegenerative diseases, neovascularization and genetic disorders. Signalling by FGF is mainly mediated through binding to both highaffinity tyrosine kinase receptors and low-affinity heparan sulfate proteoglycans associated with plasma membrane and extracellular matrix. Matrix proteoglycans appear to act as a storage depot or sink for FGF, whereas membrane proteoglycans function as co-receptors and facilitate signalling by the high-affinity receptors. Four genes encoding tyrosine kinase receptors have been identified, but alternative splicing produces multiple isoforms, some of which are putative secreted receptors. Signal transduction of tyrosine kinase receptors involves dimerization and autophosphorylation which mediate the activation of a myriad of signalling proteins and second-messengers, leading to changes in gene expression. However, there is mounting evidence to suggest that these receptors may not be sufficient for all aspects of FGF signalling. The fact that the nuclear localization of $\mathrm{FGF}_{1}, \mathrm{FGF}_{2}$ and $\mathrm{FGF}_{3}$ is found to be regulated in parallel with mitogenesis and differentiation, indeed, supports also a potential role for FGF in the direct regulation of transcription, replication or other nuclear events.

\section{Accès à la base de données internationale en immunogénétique : IMGT/LIGM-DB}

La base de données IMGT/LIGM-DB (Immunoglobulines et Récepteurs T) qui, avec HLA-DB (Julia Bodmer, ICFR, Londres), appartient à la base de données internationale ImMunoGeneTics IMGT, est accessible, depuis le 10 juillet 1995, sur le serveur WWW du CNUSC (http://imgt.cnusc. fr:8104). IMGT/LIGM-DB contient à ce jour plus de 9000 séquences (6860 séquences d'immunoglobulines et 2410 de récepteurs T) de 61 espèces différentes. Les fichiers à plat des séquences annotées (900) sont accessibles sur les serveurs ftp anonyme (ftp.ebi.ac.uk/pub/ databases/imgt) et WWW (ftp://ftp.ebi.ac.uk/pub/databases/imgt) d'EMBL-EBI, depuis le 24juin 1995.

Contact :

Pr. Marie-Paule Lefranc, Coordinateur de IMGT, Tél. : 67.61.36.34., Fax : 67.04.02.31/45. E-mail : lefranc@ligm.crbm.cnrs-mop.fr 